\title{
ENDF/B-VII.0, JEFF-3.1 and JENDL-4 Iron and Water Cross Sections Analysis Using the PCA-REPLICA Shielding
}

\author{
O. Allaoui $^{1, *}$, T.El Bardouni ${ }^{1}$, El M. Chakir ${ }^{2}$, H. Boukhal ${ }^{1}$, M. Kaddour $^{1}$, B.El Bakkari ${ }^{1,3}$, \\ M.Zoubair $^{1}$, S. El Ouahdani ${ }^{1}$ \\ ${ }^{1}$ ERSN-LMR, Department of Physics, Faculty of Sciences, POB 2121, Tetuan, Morocco \\ ${ }^{2}$ EPTN-LPMR, Faculty of Sciences, University Ibn Tofail, Kénitra, Morocco \\ ${ }^{3}$ Reactor Operation Unit (UCR), National Centre of Sciences, Energy and Nuclear Techniques (CNESTEN/CENM), \\ POB 1382, Rabat, Morocco \\ *Corresponding Author:ouassima_23@hotmail.com
}

Copyright $(2014$ Horizon Research Publishing All rights reserved.

\begin{abstract}
An accurate calculation of neutrons penetration through the side-shield of a Pressurized Water Reactor (PWR) is important for the estimation of nuclear heating in the thermal shields and in the Reactor Pressure Vessel (RPV), also for estimate the leakage of the particles in the reactor cavity. The cross section data and the calculation method have an important role in this kind of problems. For this object the PCA-REPLICA benchmark where the neutron transports through typical PWR were performed at AEA technology Winfrith, UK (United Kingdom). This work aims to contribute validation of the method of calculation and analyse the cross sections, for Iron $\left({ }^{56} \mathrm{Fe}\right)$ and water $\left({ }^{1} \mathrm{H}\right.$ and ${ }^{16} O$ ), presented by: ENDF/B-VII.0, JEFF-3.1 and JENDL-4 libraries, for the PCA-REPICA benchmark, using the Monte Carlo approach by the mean of MCNPX code. The benchmark analysis was made based on the calculated-to-measured $(\mathrm{C} / \mathrm{M})$ dosimetry reaction rates of ${ }^{115} \operatorname{In}\left(n, n^{\prime}\right){ }^{15 m_{I n}} \quad, \quad{ }^{32} S\left(n, n^{\prime}\right)^{32} P$ and ${ }^{103} R h\left(n, n^{\prime}\right){ }^{103 m_{R h}}$ monitors, at different depth in a water/iron shield reproducing the ex-core radial geometry of a PWR. The calculations of PCA-REPLICA experiment showed us that calculation method is effective for the protection study of the REP. And generally, the average $\mathrm{C} / \mathrm{M}$ ratios obtained for the three detectors are reasonably good when the uncertainties of the measurements are taken into account.
\end{abstract}

Keywords Pressurized Water Reactor, PCA-REPLICA Benchmark, MCNP, Cross section, NJOY99, Validation, Variance Reduction

\section{Introduction}

The pressure vessel is an important component of the nuclear reactor, the knowledge of the fluence of neutron in this part of the reactor during its operation and their associated effects is a key issue [1]. The calculations of these types of problems require a better method of calculation and good knowledge of the cross sections of the principal materials in protection studies of nuclear reactors, such as the Iron and the water.

Despite the fact that iron and water are largely used in the nuclear industry, there are still large uncertainties associated with their nuclear data. Enhancing the cross section data of these materials is indispensable to improve shielding calculations.

To estimate the nuclear heating in the RPV and in the thermal shields as well as the leakage of particles in the reactor cavity, an accurate calculation of neutron penetration through the side-shield of a PWR is required.

This work focus on the analysis of cross sections data of ${ }^{56} \mathrm{Fe},{ }^{1} \mathrm{H}$ and ${ }^{16} \mathrm{O}$ isotopes of the more recent data libraries: ENDF/B-VII.0[2], JEFF-3.1[3] and JENDL-4[4].These isotopes where chosen because they are considered as major components for constituting the shielding structural. For this analysis, a 3-D model of the PCA-REPLICA [5,6] benchmark experiment performed at the ASPIS shielding facility of the NESTOR low flux experimental reactor at AEA technology Winfrtith [7,8], is established by the mean of the continuous energy Monte Carlo code MCNPX [9] which allows simulating all types of interactions and using point-wise cross sections. The principal goal $[5,6]$ of the PCA-REPLICA experiment is the acquisition of benchmark-quality integral neutron data to test shielding transport methods and cross section data used in the calculation of the ex-core fluxes in LWR. Cross sections data from the ENDF/B-VII.0, distributed with the MCNPX code, are used for all the rest of the benchmark structural materials, while only ${ }^{56} \mathrm{Fe},{ }^{1} \mathrm{H}$ and ${ }^{16} \mathrm{O}$ cross sections data were processed with JEFF-3.1 and JENDL-4 libraries using the processing nuclear data processing system NJOY99 [10] 
updated to its patch file "up364". Those isotopes were investigated to estimate the effects on the benchmark calculation results.

The PCA-REPLICA experiment was performed to measure the detector reaction rates at different depths in a water/iron shield reproducing the ex-core radial geometry of a PWR. Calculations were carried out using the threshold reaction rate detectors ${ }^{115_{I n}\left(n, n^{\prime}\right)}{ }^{115 m_{I n}},{ }^{32} S\left(n, n^{\prime}\right)^{32} P$ and ${ }^{103} R h\left(n, n^{\prime}\right)^{103 m_{R h}}$ located in 10 different positions. The threshold energies are $0.01,0.3$ and $2 \mathrm{MeV}$, respectively. The detectors cross section data were taken from the International Reactor Dosimetry File IRDF-2002 [11], and they have been processed into libraries suitable for use with the MCNP code using the NJOY system.

Several Variance Reduction techniques [12,13] are available by the MCNP code such as: Transport cut-offs, weight window, Russian roulette and phase space that seems to be appropriate to reduce enormously the computing time. Some of these VR techniques have been used in the frame of this study. The statistical errors obtained were found to be acceptable in all the measurements positions even for those far from the source.

\section{Materials and Methods}

For the purpose of developing our 3-D model of the benchmark study, we have used the Monte Carlo N-particle transport code MCNPX which allows describing the real geometry and materials composition. This leads to minimize the number of approximations and to highlight the errors linked to nuclear data libraries.

\subsection{Shielding Benchmark Experiment}

\subsubsection{Description of the PCA-REPLICA Benchmark Experiment}

The analysed benchmark experiment PCA-REPLICA was the preliminary experiment of the NESTOR Shielding and Dosimetry Improvement Programme (NESDIP) [6], [14-16] and it was carried out in the ASPIS facility of the NESTOR low-flux experimental reactor in the UK Atomic Energy Establishment of Winfrith [17].

The PCA-REPLICA experimental facility duplicated exactly 12/13 geometrical configuration of the Oak Ridge PCA (Pool Critical Assembly) experiment at ORNL (Oak Ridge National Laboratory) (12 and $13 \mathrm{~cm}$ of water respectively between the core and thermal shield and the RPV with the exception that the reactor source was replaced by a rectangular fission-plate to provide a well characterized neutron source, to investigate further the discrepancies between measurements and calculations observed in the PCA studies. During the experiment the rectangular fission-plate was irradiated by the NESTOR reactor $(30 \mathrm{~kW} \max$ power) through a graphite thermal column, of total thickness $43.91 \mathrm{~cm}$, in the ASPIS shielding facility, and it is constructed U-235 enriched to $93 \%$ surrounded by an aluminum layer.

The PCA-REPLICA shielding array is shown schematically in Figure 1.

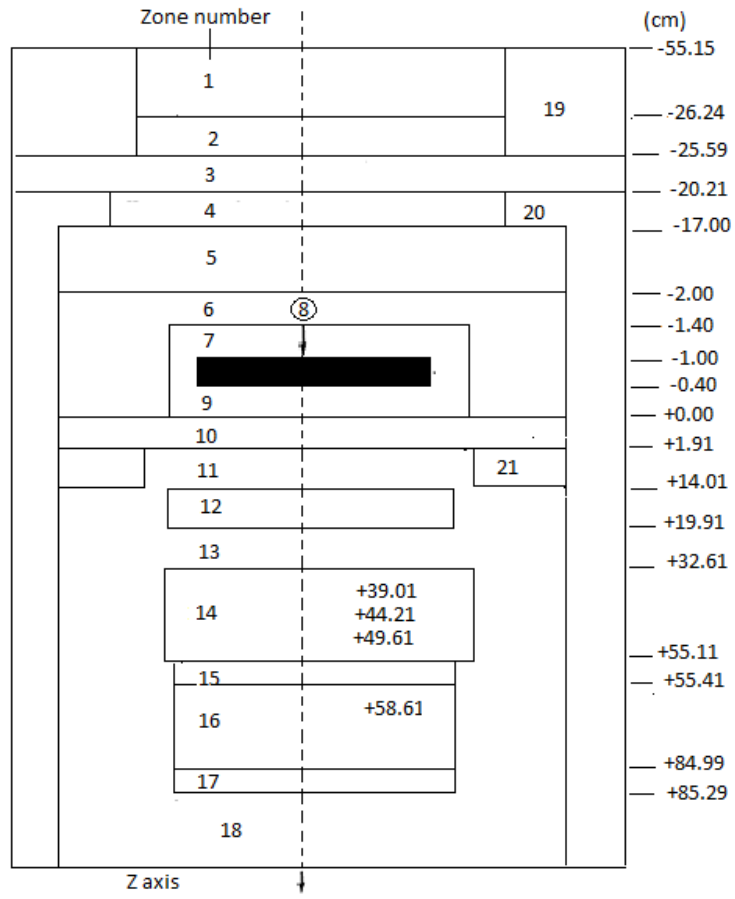

Figure 1. Layout of the 12/13 PCA-REPLICA configuration for calculation

The Materials and the zones numbers which corresponds along the $\mathrm{Z}$ axis of the PCA-REPLICA is shown in table. 1

Table.1. Materials and zones numbers along $\mathrm{Z}$ Axis for the PCA-REPLICA experiment.

\begin{tabular}{|cc|}
\hline MATERIAL & ZONE NUMBER \\
\hline Graphite & $1,5$. \\
\hline Aluminium & $2,4,7,8$ (Fission Plate), $9,10,15,17$. \\
\hline Void (Air) & $3,6,16$. \\
\hline Water & $11,13,18$. \\
\hline Stainless Steel & 12. \\
\hline Mild Steel & $14,19,20$. \\
\hline Concrete & 21. \\
\hline
\end{tabular}

It was arranged in a large steel tank filled (square section; side $180.0 \mathrm{~cm}$ ) filled with water and surrounded by a thick concrete shield. After the first water gap $(12.1 \mathrm{~cm})$, there was the stainless steel thermal shield (TS) simulator $(5.9 \mathrm{~cm}$ thick) and the second water gap $(12.7 \mathrm{~cm})$. Then the mild steel RPV simulator (thickness $\mathrm{T}=22.5 \mathrm{~cm}$ ) was located and tightly connected with a void box made of a thin layer of aluminium, simulating the cavity (thickness $=29.58 \mathrm{~cm}$ ) between the RPV and the biological shield in a real PWR. The axial dimensions of the experimental components are given in Table. 2 . 
Table.2. Materials and dimensions along $\mathrm{X}$ and $\mathrm{Y}$ lateral Axes for the PCA-REPLICA experiment.

\begin{tabular}{|c|c|c|c|}
\hline Zone & Material & Section & Dimensions (cm) \\
\hline 1 & Carbon & Square & $119 \times 119$ \\
\hline 2 & Aluminum & Square & $119 \times 119$ \\
\hline 3 & Void & Square & $185 \times 185$ \\
\hline 4 & Aluminum & Circular & $\mathrm{r}=56,06$ \\
\hline 5 & Carbon & Square & $180 \times 180$ \\
\hline 6 & Void & Square & $180 \times 180$ \\
\hline 7 & Aluminum & Rectangular & $68,5 \times 47,5$ \\
\hline 8 & Fuel & Rectangular & $63,5 \times 40,2$ \\
\hline 9 & Aluminum & Rectangular & $180 \times 180$ \\
\hline 10 & Aluminum & Square & $180 \times 180$ \\
\hline 11 & Water & Square & $180 \times 180$ \\
\hline 12 & Stainless steel & Square & $68,5 \times 68,5$ \\
\hline 13 & Water & Square & $180 \times 180$ \\
\hline 14 & Mild Steel & Square & $68,5 \times 68,5$ \\
\hline 15 & Aluminum & Square & $60 \times 60$ \\
\hline 16 & Void & Square & $59,4 \times 59,4$ \\
\hline 17 & Aluminum & Square & $60 \times 60$ \\
\hline 18 & Water & Square & $180 \times 180$ \\
\hline 19 & Stainless steel & Square & $180 \times 180$ \\
\hline 20 & Stainless steel & Square & $185 \times 185$ \\
\hline 21 & Concrete & Square & $285 \times 285$ \\
\hline
\end{tabular}

All these elements were perfectly orthogonally aligned and centred along the imaginary line (horizontal axis $\mathrm{z}$ ) passing through the centre of the fission plate. Along the $z$ axis, three types of thresholds detectors give the gave the integral measurements (reaction rates) at different depth in a water/iron shield reproducing the ex-core radial geometry of a PWR ${ }^{103} R h\left(n, n^{\prime}\right)^{103 m_{R h}} \quad{ }^{115_{I n}\left(n, n^{\prime}\right)^{115 m_{I n}}}$ and ${ }^{32} S\left(n, n^{\prime}\right){ }^{32} P$. These detectors were located at 10 different positions: in the water gaps 1.91, 7.41, 12.41, 14.01, 19.91, $25.41,30.41 \mathrm{~cm}$ from the fission plate $(\mathrm{Rh}$ measurements only) and at $1 / 4$ and $3 / 4$ thickness of the RPV and in the void box $(\mathrm{Rh}, \mathrm{In}, \mathrm{S})$. The threshold energies are $0.01,0.3$ and $2 \mathrm{MeV}$, respectively.

\subsubsection{MCNP modeling of PCA-REPLICA benchmark}

These experiments have very well defined sources, geometries and material compositions so that they can be modelled in 3-D with very little uncertainty using the Monte Carlo code MCNP. Figures 2 and 3 represent the radial shield and the source plate modelled with MCNP, respectively.

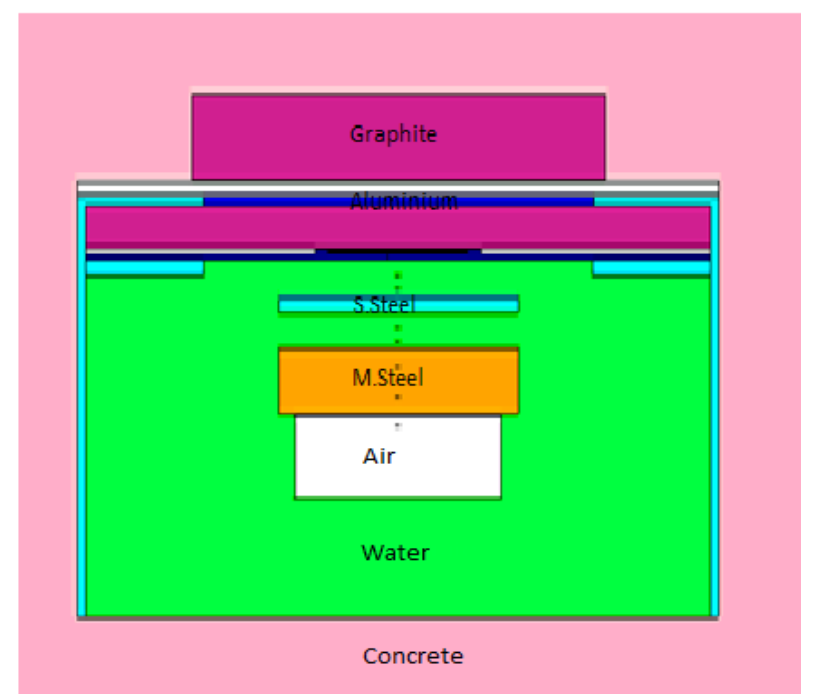

Figure 2. MCNP model for the radial shield of benchmark PCA-REPLICA

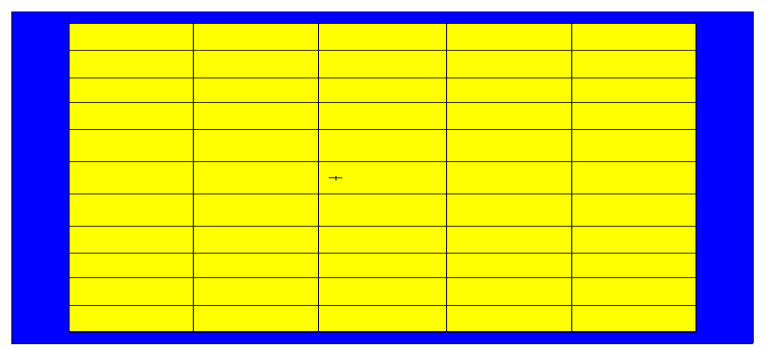

Figure 3. MCNP model for the source plate geometry

The MCNP code in its extended version MCNPX is a very powerful tool to represent the flux cartography of the modelled geometry, through the MESH card.

Figure 4 and 5 give the flux cartography in 2-D and 3-D obtained using the MESH card on the shield of the PCA-REPLICA benchmark and in the fission plate, respectively.

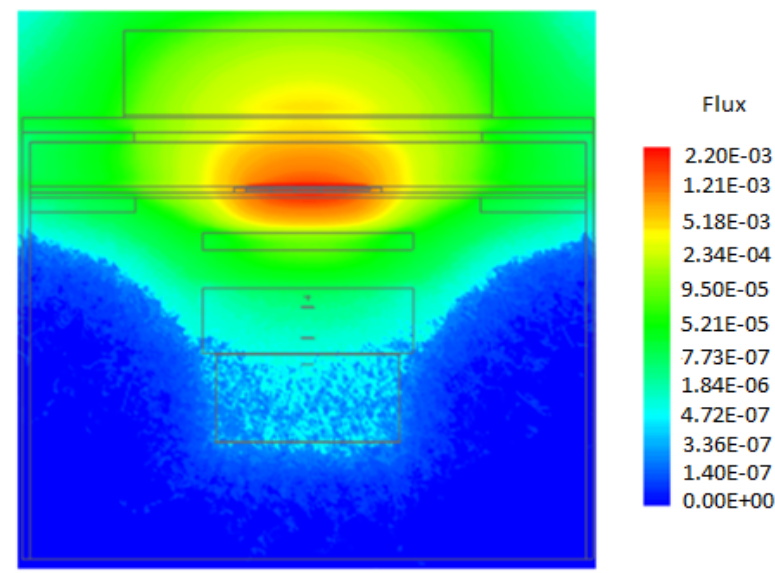

Figure.4. Density distribution of the neutron flux according $\mathrm{XZ}$ in the shield of the PCA-REPLICA 


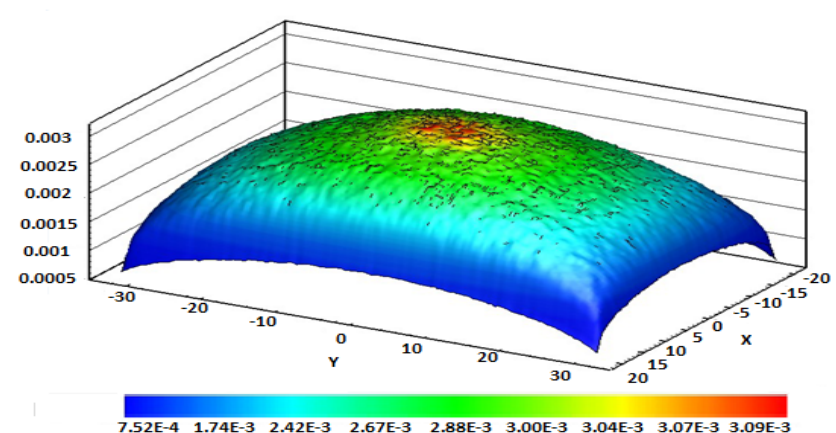

Figure.5. The distribution of the neutron flux density at the fission plate in 3-D.

It is clearly seen from these figures that the highest flux density is obtained at the centre of the fission plate and decreases when it moves radially and axially towards the extremities.

\subsection{Continuous Cross-Section Library Generation}

The process to construct the continuous cross-section data libraries from the data source files is typically performed by the NJOY system code. In this study, the NJOY99 [10] system with its latest update file "up364", has been used to process the source evaluated nuclear data files into libraries suitable for use with the MCNP code. The procedure to process point-wise cross-sections by each module of NJOY is shown in Figure. 6.

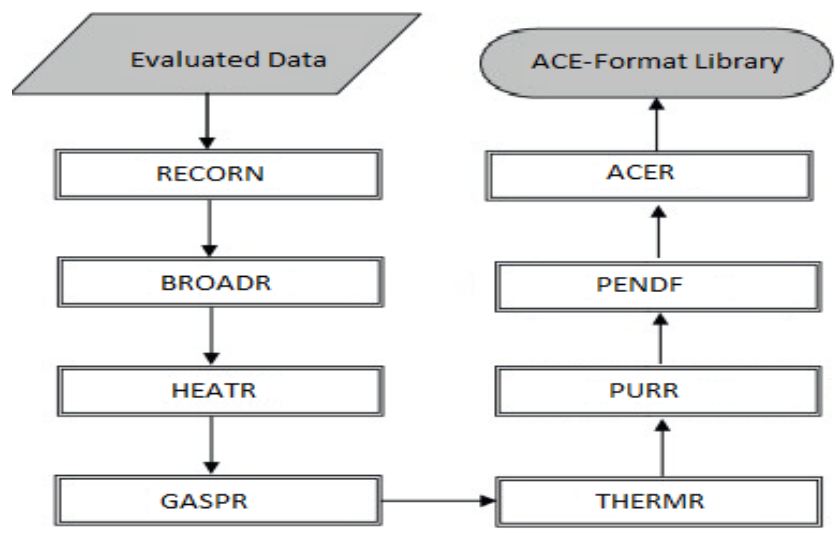

Figure.6. Flow diagram of NJOY99 processing for ACE format library construction.

The principal advantage of NJOY is its most general-purpose applicability and comprehensive capability to process data in the recent ENDF format. It takes the basic data from the nuclear data library and converts them into forms needed for applications. Resonance cross-sections are constructed using a method of choosing the energy grid that incorporates control over the number of grid points generated for some materials. Summation cross-sections are reconstructed from their parts. The resulting point-wise cross-sections are written onto a "point-ENDF" (PENDF) file for future use. BROADR reads a PENDF file and
Doppler-broadens the data. After broadening and thinning, the summation cross-sections are again reconstructed from their parts. The results are written onto a new PENDF file for future use. HEATR computes energy-balance heating and damages energy using reaction kinematics or applying conservation of energy. The ENDF photon production files can be used in this step, when available. GASPR module goes through all of the reactions given in an ENDF-format evaluation, determines which charged particles would be produced by the reaction, and adds up the particle yield times the reaction cross-section to produce the desired gas production cross-sections. THERMR produces point-wise cross- section in the thermal range. Energy-to-energy incoherent inelastic scattering matrices can be computed for free-gas scattering or for bound scattering using a precomputed scattering law in ENDF file. PURR is used to prepare unresolved-region probability tables. ACER prepares cross-sections and scattering laws in ACE format (a compact ENDF format) for the MCNP computer code. All the cross-sections are represented on a union grid for linear interpolation by taking advantage of the representation used in RECONR and BROADR.

Finally, the $\mathrm{S}(\alpha, \beta)$ thermal scattering cross-sections of bound nuclei ( $\mathrm{H}$ in $\mathrm{H}_{2} \mathrm{O}, \mathrm{C}$ in graphite, the Aluminum and ${ }^{56} \mathrm{Fe}$ in the steel) were taken directly from the standard MCNP cross-section library respecting the shielding characteristics of PCA-REPLICA. These thermal scattering data are essential to accurately model the neutron interactions at energies below $\sim 4 \mathrm{eV}$ [9].

\subsection{Overview of Variance Reductions Techniques}

The Variance reduction techniques are important elements of any Monte Carlo code and may be different for various applications and geometries. These techniques reduce the time of particle simulations and improve the speed of the code. There are four main categories of variance reduction techniques, detailed in the following paragraph:

Truncation Methods: are the simplest variance reduction methods. They speed up calculations by truncating parts of space that do not contribute significantly to the solution. The simplest example concerns geometry truncation in which unimportant parts of the geometry are simply not modelled [18]. The specific truncation methods available in MCNPX are the energy cutoff, weight cutoff and time cutoff. In this work, we are interested in energy cutoff (CUT card) as we have threshold detectors.

Population Control Methods: These methods artificially increase the number of particles in spatial or energy regions that are important or decrease this number in the case it doesn't contribute to the score tally. In MCNPX, the specific methods of population control that we used are Geometry splitting and Russian roulette (IMP card).

Modified Sampling Methods: These methods artificially increase the likelihood of events increasing the particle of interest probability to reach the tally region. 
Partially-Deterministic Methods: Are the most complicated class of variance reduction methods. They circumvent the normal random walk process by using deterministic-like techniques, such as next event estimators, or by controlling the random number sequence [19].

An excellent overview of classic MCNP VR techniques was given by Booth [13]. In our calculation the statistical errors obtained are found to be acceptable in all positions of measurement even for the points which are far from the source, through the use of some VR techniques such as: the phase space, energy cut-off (CUT card) as we have threshold detectors, the Geometry splitting and Russian roulette (IMP card).

\section{Results and Discussion}

The reaction rates for each detector and for different nuclear data files of the ${ }^{56} \mathrm{Fe},{ }^{16} \mathrm{O}$ and ${ }^{1} \mathrm{H}$ isotopes have been calculated with the MCNP code for several positions.

Tables 3-5 show the calculated-to-measured (C/M) reaction rates ratios calculated for $12 \mathrm{~cm}, 13 \mathrm{~cm}$ water gap as well as RPV and cavity and also the average values obtained for the threshold detectors used in the PCA-REPLICA benchmark experiment, for each nuclear data library used in this study.

The ${ }^{103} R h\left(n, n^{\prime}\right)^{103 m} R h$ calculated reaction rates (table 3) are well predicted in the RPV simulator within a maximum discrepancy of $5 \%$. However the results are worse in the water gaps and the cavity, In this positions the reaction rates are below experimental values for all the data files, this underestimation is more important for the last point of each water gap, in the range between $7 \%$ and $15 \%$. After having crossed a steel plate (region 12 in the figure 3), this underprediction is less important ( 3 of $4 \%$ ).

The average ratios obtained for this detector are relatively constant among the three different nuclear data libraries (with slight discrepancies within $\sim 2 \%$ ), that means the difference evaluations does not affect the acceptability of the ${ }^{103} R h\left(n, n^{\prime}\right){ }^{103 m} R h$ detector.

Generally a good agreement between calculation and measurement for all the nuclear data library was evident as illustrated in table 4, which represent the calculated-to-measured $(\mathrm{C} / \mathrm{M})$ ratios and the average of the dosimetry reaction rates obtained for the ${ }^{115} \operatorname{In}\left(n, n^{\prime}\right)^{115 m}$ In detector. The results obtained for ${ }^{1} H$ show small discrepancies, within 1\%, among the two different nuclear data libraries used in the calculation. The effects of using different evaluated nuclear data files were relatively high for the ${ }^{56} \mathrm{Fe}$ and ${ }^{16} \mathrm{O}$ data; however, the differences between the cross section of the ${ }^{1} H$ from JENDL-4 and JEFF-3.1 are relatively small. The results are significantly improved using the nuclear data of ${ }^{16} O$ from JENDL-4 compared to those derived from JEFF-3.1.

The ${ }^{1} \mathrm{H}$ data from ENDFB/VII.0 have been substituted with those of JENDL-4, the result is compared for this detector.

Table.3. . Calculated-to-measured ratio of the dosimetry reaction rates for the ${ }^{103} R h\left(n, n^{\prime}\right){ }^{103 m} R h$ detector.

\begin{tabular}{|c|c|c|c|c|c|c|c|c|}
\hline \multicolumn{9}{|c|}{${ }^{103} R h\left(n, n^{\prime}\right){ }^{103 m} R h$} \\
\hline Reference & $\begin{array}{c}\text { Distance } \\
\text { from Fission } \\
\text { plate }(\mathrm{cm})\end{array}$ & $\begin{array}{c}\text { ENDF/B-VI } \\
\text { I.0 }\end{array}$ & $\begin{array}{c}{ }^{56} \mathrm{Fe} \text { from } \\
\text { JENDL.4 }\end{array}$ & $\begin{array}{c}{ }^{56} \mathrm{Fe} \text { from } \\
\text { JEFF-3.1 }\end{array}$ & $\begin{array}{l}{ }^{1} H \text { from } \\
\text { JENDL.4 }\end{array}$ & $\begin{array}{l}{ }^{1} H \text { from } \\
\text { JEFF-3.1 }\end{array}$ & $\begin{array}{c}{ }^{16} O \text { from } \\
\text { JENDL.4 }\end{array}$ & $\begin{array}{c}{ }^{16} O \text { from } \\
\text { JEFF-3.1 }\end{array}$ \\
\hline \multirow{4}{*}{$\begin{array}{c}12 \mathrm{~cm} \text { water } \\
\text { gap }\end{array}$} & 1.91 & $\begin{array}{c}0.96( \pm 0.2) \\
(a)\end{array}$ & $0.96( \pm 0.20)$ & $0.96( \pm 0.20)$ & $0.96( \pm 0.20)$ & $0.96( \pm 0.20)$ & $0.96( \pm 0.20)$ & $0.96( \pm 0.20)$ \\
\hline & 7.41 & $0.89( \pm 0.4)$ & $0.88( \pm 0.33)$ & $0.88( \pm 0.34)$ & $0.88( \pm 0.33)$ & $0.89( \pm 0.37)$ & $0.88( \pm 0.35)$ & $0.88( \pm 0.34)$ \\
\hline & 12.41 & $0.90( \pm 0.63)$ & $0.90( \pm 0.54)$ & $0.90( \pm 0.54)$ & $0.90( \pm 0.54)$ & $0.90( \pm 0.58)$ & $0.90( \pm 0.51)$ & $0.90( \pm 0.52)$ \\
\hline & 14.01 & $0.86( \pm 0.75)$ & $0.87( \pm 0.73)$ & $0.87( \pm 0.75)$ & $0.86( \pm 0.62)$ & $0.85( \pm 0.61)$ & $0.86( \pm 0.72)$ & $0.86( \pm 0.64)$ \\
\hline \multirow{3}{*}{$\begin{array}{c}13 \mathrm{~cm} \text { water } \\
\text { gap }\end{array}$} & 19.91 & $0.95( \pm 1.5)$ & $0.96( \pm 0.96)$ & $0.97( \pm 1.00)$ & $0.97( \pm 0.93)$ & $0.97( \pm 1.05)$ & $0.96( \pm 0.95)$ & $0.96( \pm 0.92)$ \\
\hline & 25.41 & $0.95( \pm 1.01)$ & $0.94( \pm 1.02)$ & $0.95( \pm 1.01)$ & $0.95( \pm 1.01)$ & $0.94( \pm 1.01)$ & $0.95( \pm 1.02)$ & $0.95( \pm 1.01)$ \\
\hline & 30.41 & $0.94( \pm 0.88)$ & $0.94( \pm 0.89)$ & $0.95( \pm 0.88)$ & $0.94( \pm 0.88)$ & $0.93( \pm 0.89)$ & $0.95( \pm 0.88)$ & $0.94( \pm 0.88)$ \\
\hline \multirow{2}{*}{$\begin{array}{c}\text { RPV: T/4 } \\
3 \mathrm{~T} / 4\end{array}$} & 39.01 & $0.98( \pm 0.31)$ & $0.96( \pm 0.31)$ & $0.99( \pm 0.31)$ & $0.98( \pm 0.32)$ & $0.96( \pm 0.32)$ & $0.99( \pm 0.31)$ & $0.97( \pm 0.31)$ \\
\hline & 49.61 & $0.99( \pm 0.32)$ & $0.98( \pm 0.35)$ & $1.05( \pm 0.32)$ & $0.99( \pm 0.32)$ & $0.98( \pm 0.32)$ & $1.01( \pm 0.33)$ & $0.99( \pm 0.36)$ \\
\hline Cavity & 58.61 & $0.94( \pm 0.46)$ & $0.91( \pm 0.37)$ & $0.98( \pm 0.45)$ & $0.94( \pm 0.41)$ & $0.93( \pm 0.45)$ & $0.95( \pm 0.42)$ & $0.92( \pm 0.38)$ \\
\hline Average & & 0.94 & 0.93 & 0.95 & 0.94 & 0.93 & 0.94 & 0.93 \\
\hline
\end{tabular}

\footnotetext{
${ }^{(a)}$ The uncertainty (\%) of the measurement
} 
Table.4. Calculated-to-measured ratio of the dosimetry reaction rates for the ${ }^{115} \operatorname{In}\left(n, n^{\prime}\right)^{115 m} \operatorname{In}$ detector.

\begin{tabular}{|c|c|c|c|c|c|c|c|c|}
\hline \multicolumn{9}{|c|}{$\left.{ }^{115_{I n}\left(n, n^{\prime}\right)}\right)^{115 m_{I n}}$} \\
\hline Reference & $\begin{array}{l}\text { Distance from } \\
\text { Fission plate } \\
\text { (cm) }\end{array}$ & ENDF/B-VII.0 & $\begin{array}{c}\quad 56_{F e} \\
\text { from } \\
\text { JENDL.4 }\end{array}$ & $\begin{array}{c}{ }^{56} F e \text { from } \\
\text { JEFF-3.1 }\end{array}$ & $\begin{array}{l}{ }^{1} H \text { from } \\
\text { JENDL.4 }\end{array}$ & $\begin{array}{l}{ }^{1} H \text { from } \\
\text { JEFF-3.1 }\end{array}$ & $\begin{array}{l}{ }^{16} O \text { from } \\
\text { JENDL.4 }\end{array}$ & $\begin{array}{c}{ }^{16} O \text { from } \\
\text { JEFF-3.1 }\end{array}$ \\
\hline RPV: T/4 & 39.01 & $1.00( \pm 0.23)$ & $0.98( \pm 0.24)$ & $1.02( \pm 0.23)$ & $1.00( \pm 0.23)$ & $\begin{array}{c}0.98 \\
( \pm 0.23) \\
\end{array}$ & $\begin{array}{c}1.02 \\
( \pm 0.23) \\
\end{array}$ & $0.99( \pm 0.23)$ \\
\hline $3 T / 4$ & 49.61 & $0.96( \pm 0.18)$ & $0.94( \pm 0.18)$ & $1.01( \pm 0.18)$ & $0.96( \pm 0.18)$ & $\begin{array}{c}0.95 \\
( \pm 0.18) \\
\end{array}$ & $\begin{array}{c}0.98 \\
( \pm 0.18)\end{array}$ & $0.95( \pm 0.18)$ \\
\hline Cavity & 58.61 & $0.98( \pm 0.21)$ & $0.96( \pm 0.21)$ & $1.04( \pm 0.20)$ & $0.98( \pm 0.21)$ & $\begin{array}{c}0.97 \\
( \pm 0.21)\end{array}$ & $\begin{array}{c}1.00 \\
( \pm 0.21)\end{array}$ & $0.98( \pm 0.21)$ \\
\hline Average & & 0.98 & 0.96 & 1.02 & 0.98 & 0.97 & 1.00 & 0.97 \\
\hline
\end{tabular}

Table.5. Calculated-to-measured ratio of the dosimetry reaction rates for the ${ }^{32} S\left(n, n^{\prime}\right)^{32} P$ detector.

\begin{tabular}{|c|c|c|c|c|c|c|c|c|}
\hline \multicolumn{9}{|c|}{${ }^{32} S\left(n, n^{\prime}\right)^{32} P$} \\
\hline Reference & $\begin{array}{c}\text { Distance from } \\
\text { Fission plate } \\
\text { (cm) }\end{array}$ & ENDF/B-VII.0 & $\begin{array}{c}{ }^{56} \mathrm{Fe} \text { from } \\
\text { JENDL.4 }\end{array}$ & $\begin{array}{c}{ }^{56} \mathrm{Fe} \text { from } \\
\text { JEFF-3.1 }\end{array}$ & $\begin{array}{l}{ }^{1} \mathrm{H} \text { from } \\
\text { JENDL.4 }\end{array}$ & $\begin{array}{c}{ }^{1} \mathrm{H} \text { from } \\
\text { JEFF-3.1 }\end{array}$ & $\begin{array}{c}{ }^{16} O \text { from } \\
\text { JENDL.4 }\end{array}$ & $\begin{array}{c}{ }^{16} O \text { from } \\
\text { JEFF-3.1 }\end{array}$ \\
\hline RPV: T/4 & 39.01 & $0.95( \pm 0.5)$ & $0.93( \pm 0.52)$ & $0.97( \pm 0.5)$ & $0.95( \pm 0.51)$ & $0.94( \pm 0.51)$ & $1.00( \pm 0.5)$ & $0.94( \pm 0.51)$ \\
\hline $3 T / 4$ & 49.61 & $0.98( \pm 0.33)$ & $0.96( \pm 0.39)$ & $1.02( \pm 0.37)$ & $0.98( \pm 0.39)$ & $0.97( \pm 0.39)$ & $1.03( \pm 0.38)$ & $0.97( \pm 0.39)$ \\
\hline Cavity & 58.61 & $1.04( \pm 0.33)$ & $1.05( \pm 0.48)$ & $1.10( \pm 0.46)$ & $1.04( \pm 0.47)$ & $1.03( \pm 0.47)$ & $1.05( \pm 0.46)$ & $1.04( \pm 0.47)$ \\
\hline Average & & 0.99 & 0.98 & 1.03 & 0.99 & 0.98 & 1.02 & 0.98 \\
\hline
\end{tabular}

The results for the ${ }^{32} S\left(n, n^{\prime}\right)^{32} P$ reaction rate are presented in table 4 . The average $\mathrm{C} / \mathrm{M}$ ratios for this reaction are generally well maintained, within small discrepancies of $3 \%$. For this higher threshold detector (response domain of the ${ }^{32} S\left(n, n^{\prime}\right)^{32} P$ is superior to $2 \mathrm{MeV}$ ), the underpredictions of the calculated activities tend to increase with the neutron penetration depth (see Table. 5).

The calculations using JEFF-3.1 for the ${ }^{56} \mathrm{Fe}$ show a maximum overprediction of $10 \%$ in the cavity for the ${ }^{32} S\left(n, n^{\prime}\right){ }^{32} P$ detector. For this detector, calculations have also been performed using the data library based on JEFF-3.1 and JENDL-4 data for the ${ }^{56} \mathrm{Fe},{ }^{16} \mathrm{O}$ and ${ }^{1} \mathrm{H}$. The calculated results using the ${ }^{1} \mathrm{H}$ from JEFF-3.1 are approximately similar to those from JENDL-4 for the same data; however, the different between the results by JENDL-4 and JEFF-3.1 for the ${ }^{56} \mathrm{Fe}$ and ${ }^{16} O$ data are somewhat larger. An underprediction with the cross section of the ${ }^{16} O$ from JEFF3.1, (2\%) becoming an overprediction with JENDL-4. The average $\mathrm{C} / \mathrm{M}$ dosimetry reaction rates with the different nuclear data files of the ${ }^{56} \mathrm{Fe}$ shows that the JEFF.3.1 over-predicted the attenuation, while a calculation with JENDL-4 underpredicted the transmission of neutrons compared to the other library (the differences between the two libraries are 5\%).

The calculated results using ENDF/B-VII.0 and ENDF/B-VII.0 with the cross section of ${ }^{1} H$ showed a large similar.

\section{Conclusion}

The calculations of PCA-REPLICA experiment performed in the ASPIS shielding facility of the UK, showed us that calculation method (Monte Carlo) is effective for the protection study of the REP. Generally in the PCA-REPLICA benchmark, the average $\mathrm{C} / \mathrm{M}$ ratios obtained for the three detectors are reasonably good when the uncertainties of the measurements are taken into account. The effects of using different evaluated nuclear data files were relatively high for ${ }^{56} \mathrm{Fe}$ and ${ }^{16} \mathrm{O}$ data. The average

$\mathrm{C} / \mathrm{M}$ ratios of the dosimetry reaction rates evaluated at all the detector positions of the PCA-REPLICA experiment with the ENDFB/VII.0 library are similar of those with $\mathrm{H}$ from JENDL-4 while the results using ${ }^{56} \mathrm{Fe}$ from JENDL-4 showed a tendency to under-predict when compared with the other libraries.

The ENDF/B-VII.0 library is more adapted for ${ }^{32} S\left(n, n^{\prime}\right)^{32} P$ detector than for the others, it means that ENDF/B-VII.0 is adapted for high energy.

\section{Acknowledgements}

The authors would like to thank the team of Radiation and Nuclear System especially the Professor El bardouni Tarek for their great scientific advices.

REFERENCES 
[1] Song Hui Zheng : « qualification des méthodes de calculs de fluence dans les réacteurs à eau pressurisées. Amélioration du traitement des sections efficaces par la méthode des tables de probabilité ». Thèse de l'Université de Paris-Sud, numéro d'ordre 2887, Note CEA-N-2745, 1993.

[2] Chadwick et al., 2006 Chadwick, M.B., et al., 2006. ENDF/B-VII.0.0: Next Generation Evaluated Nuclear Data Library For Nuclear Science and Technology, Nuclear data Sheets,vol.107,pp.2931-3060. Online available from http://www.nndc.bnl.gov/exfor7/endf00.htm.

[3] The JEFF Team, 2006 The JEFF Team, 2006.The JEFF-3.1 library, JEFF Report 21,NEA/OECD No. 6190, 2006, ISBN92-64-02314-3. Online available from http://www.nea.fr/html/dbdata/projects/nds_jef.htm

[4] On line available from http://www.nndc.bnl.gov/sigma/ ,(july,2013)

[5] J. Butler, The NESTOR Shielding and Dosimetry Improvement Programme NESDIP for PWR Applications, PRPWG/P(82)5, Internal UKAEA Document, November 1982.

[6] J.Butler et al., "The PCA PCA-REPLICA Experiment, PART 1, Winfrith Measurements and Calculations and calculations», AEEW -R 1736, Reactor Physics Division, AEE Winfrith, Jun. 1984.

[7] W.N.McElroy Editor, LWR Pressure Vessel Surveillance Dosimetry Improvement Program: PCA Experiments and Blind Test, HEDL-TME 80-87, NUREG/CR-1861, 1981.

[8] Pescarini, M. and Borgia, M. G. JENDL-3.1 iron validation on the PCA-REPLICA (H2O/Fe) shielding benchmark experiment. Report No. RT/ERG/97/1 Bologna: (ENEA) (1997).

[9] D. B. Pelowitz, "MCNPXTM User's Manual Version 2.5.0," 2005.

[10] MacFarlane, R.E., 2002. NJOY-99 Nuclear Data Processing System [online]. On line available from http://t2.lanl.gov/codes/njoy99/index.html (update web site).

[11] O. Bersillon, L.R. Greenwood, P.J. Griffin, W. Mannhart,
H.J.Nolthenius, R. Paviotti-Corcuera, K.I. Zolotarev, E.M. Zsolnay, P.K. McLaughlin, A. Trkov, International Reactor Dosimetry File 2002 (IRDF-2002), 2006, International Atomic Energy Agency, Vienna, Austria, Technical Reports Series No. 452, ISBN 92-0-105106-9, December 2006, http://www-pub.iaea.org/MTCD/publications/PDF/TRS452 web.pdfS.

[12] RICHARD H. OLSHER, 2006. A practical look at Monte Carlo VR methods in radiation shielding. Health Physics Measurements Group, Los Alamos National Laboratory77, 94.

[13] T.E. Booth, "A Sample Problem in VR in MCNP," Los Alamos National Laboratory, LA-10363-MS (1985).

[14] M.D. Carter, I.J. Curl, P.C. Miller, A. Packwood, Light-Water Reactor Radial Shield benchmark Studies of the NESTOR Shielding and Dosimetry Improvement Programme (NESDIP), Reactor Dosimetry: Methods, Applications, and Standardization, ASTM STP 1001, Harry Farrar IV and E.P. Lippincott Editors, ASTM, Philadelphia.

[15] J. Butler, M.D. Carter, I.J. Curl, P.C. Miller, A. Packwood, S.Power, Review of the NESTOR Shielding and Dosimetry Improvement Programme (NESDIP), Reactor Dosimetry: Methods, Applications, and Standardization, ASTM STP 1001, Harry Farrar IV and E.P. Lippincott Editors, ASTM, Philadelphia 1989, pp. 295-307.

[16] P.C. Miller, A REVIEW OF LWR PRESSURE VESSEL DOSIMETRY AND ASSOCIATED SHIELDING STUDIES, Proceedings of the UKAEA/OECD-NEA 7th International Conference on Radiation Shielding, Bournemouth (UK), September 12-16, 1988, VOL. I, pp. 33-47.

[17] N.D. Carter,I .J . Curl, NESTOR Shielding and Dosimetry Improvement Programme. The ASPIS-PCA Slab Geometry Benchmarks. Blind Test Edition. AEEW-W 2329, UKAEA, Winfrith, June 1986 (Corrigenda).

[18] X-5 Monte Carlo Team MCNP, "A General Monte Carlo N-Particle Transport Code," Version 5, 2003.

[19] J. K. Shultis and R. E. Faw, “An MCNP Primer," Kansas State University, Manhattan, 2011. 